\title{
Manejo de la infección del tracto urinario en lactantes febriles: Experiencia de tratamiento antimicrobiano intravenoso ambulatorio
}

\author{
Anamaría Peña D., Tamara Viviani S., Nicole Le Corre P., \\ Viera Morales M., Constanza Montecinos B. y Cristina Gajardo S.
}

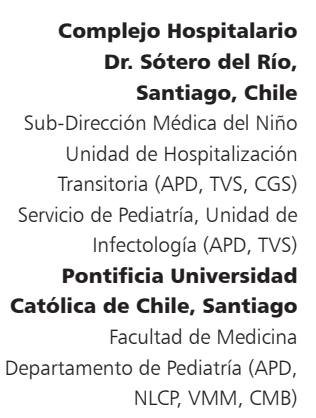

Recibido: 23 de diciembre de 2008 Aceptado: 20 de mayo de 2009

Correspondencia a: Anamaría Peña Donati anapena@med.puc.c

\section{Treatment of urinary tract infections in febrile infants: Experience of outpatient intravenous antibiotic treatment}

Objective: To describe the feasibility, effectiveness and safety of intravenous (iv) outpatient treatment in 2 to 24 month-old children with febrile urinary tract infection (UTI). Method: Children presenting to the ER, between April 2003-2005, with fever and no identifiable focus who had a diagnosis of UTI were randomized to receive iv antibiotic in the hospital or in an outpatient facility. Children were started on amikacin or ceftriaxona according to physician criteria followed by antimicrobial adjustment based on urine culture result and a later switch to an oral antimicrobial. Urine cultures were performed during and after completing the antimicrobial course. Adherence and effectiveness of antimicrobial treatment and treatment-associated complications were analyzed. Results: The study included 112 patients, 58 inpatient children and 54 outpatient children, with an average age of 7.7 months. Duration of iv treatment did not differ among groups (2.8 days (SD 1.2) $2.7+0.91$ days in inpatients vs $2.9+1.9$ days in outpatients $(\mathrm{p}=0.22)$. In $100 \%$ of outpatient children and $100 \%$ of inpatient children (overall 101/101) urine cultures were negative on day 5 . None of the children had a treatment-associated complication. Cost analysis yielded $73 \%$ of saving money (overall cost for inpatient treatment US 9,815 vs outpatient treatment US 2,650). Conclusions: Outpatient iv treatment in patients between 2 and 24 months with UTI and fever was effective, safe and of lower cost

Key words: Urinary tract infection, children, outpatient parenteral antimicrobial treatment.

Palabras clave: Infección del tracto urinario, niños, tratamiento antimicrobiano parenteral ambulatorio.

\section{Introducción}

$\mathrm{L}$ a infección del tracto urinario (ITU) corresponde a una de las patologías infecciosas más frecuentes de la infancia, presentándose en $\sim 8 \%$ de los niñas $\mathrm{y} \sim 2 \%$ de los varones bajo siete años de edad ${ }^{1-5}$. En nuestro medio, es un motivo frecuente de visita a los servicios de urgencia, alcanzando a $1,35 \%$ del total de consultas y una tasa de hospitalización de $10 \%$ en una experiencia nacional ${ }^{6}$.

En lactantes con síndrome febril sin foco, la ITU es su causa en $\sim 7,5 \%$ de los casos bajo ocho semanas de vida, $5,3 \%$ bajo un año de edad y $4,1 \%$ bajo dos años ${ }^{7,8}$. En estudios nacionales se ha reportado que en niños entre seis semanas y tres años de edad con infección bacteriana confirmada, la ITU representa el $80,2 \%$ de ellas 9 .

Algunas investigaciones han demostrado que la probabilidad de daño de parénquima renal secundario a infección urinaria varía de acuerdo a la edad, siendo mayor en niños bajo dos años, lo que justifica el tratamiento inicial apropiado en este grupo etario ${ }^{10}$.
De acuerdo a algunos autores, la hospitalización de los pacientes con ITU debería considerarse siempre en las primeras seis semanas de vida, en pacientes bajo cinco años con compromiso sistémico o hemodinámico y cuando no sea posible asegurar una buena tolerancia (vómitos, rechazo), adherencia a la terapia oral y accesibilidad al servicio de salud. Así mismo, se podría plantear un tratamiento ambulatorio en pacientes sin enfermedad sistémica en los cuales se pueda asegurar buena adherencia al tratamiento y seguimiento posterior ${ }^{3,4}$. Respecto a la elección de tratamiento iv u oral, existen algunos reportes en los cuales no se demuestran diferencias significativas en el resultado final obtenido entre ambas terapias ${ }^{11,12}$.

En los últimos años, la terapia iv ambulatoria ha sido evaluada en pacientes pediátricos con diferentes enfermedades infecciosas, tanto en niños antes sanos como en pacientes con alguna enfermedad de base. Esta modalidad ha resultado eficaz y segura, logrando a su vez reducir los costos de tratamiento. Existen algunas publicaciones que proponen esta forma de tratamiento como una buena alternativa para lactantes con ITU y 
fiebre $^{13}$. Actualmente, se dispone de antimicrobianos (ceftriaxona y amikacina) que pueden ser administrados una vez al día con demostrada eficacia y seguridad en pacientes pediátricos, sin reportarse efectos adversos de importancia ni complicaciones de la vía venosa ${ }^{14-17}$.

El Complejo Hospitalario Dr. Sótero del Río (CHDSR) cuenta con una unidad de hospitalización transitoria (UHT) a cargo del equipo de Infectología, con la infraestructura necesaria y personal entrenado para administrar tratamiento y controlar en forma periódica a pacientes con terapia parenteral.

El objetivo de este estudio fue evaluar y comparar dos grupos de lactantes febriles con ITU y tratamiento antimicrobiano iv, uno administrado con hospitalización y otro en forma ambulatoria, en cuanto a factibilidad, seguridad y eficacia. Se describen además los beneficios obtenidos y las complicaciones observadas.

\section{Material y Métodos}

Se realizó un estudio prospectivo analítico en la UHT del CHDSR, entre abril de 2003 y abril 2005. Criterios de inclusión: todos los pacientes febriles (temperatura $\geq 38^{\circ} \mathrm{C}$, en una toma), entre dos meses y dos años de edad, cuyo urocultivo a las 48 horas confirmara diagnóstico de ITU (urocultivo por cateterismo vesical con recuento mayor a $10.000 \mathrm{ufc} / \mathrm{ml}$ de orina) y que cumplieran con las siguientes condiciones: ausencia de compromiso hemodinámico, sin antecedentes de ITU o malformación de vía urinaria y en quienes no se podía asegurar la adherencia a la terapia oral por mala tolerancia. No se hizo ecografía renal previa a su inclusión en el estudio. Criterios de exclusión: aquellos pacientes con antecedente de inmunodeficiencia, patología crónica conocida y en quienes el tutor no firmó el consentimiento informado

Se ofreció tratamiento iv ambulatorio a todo paciente con síndrome febril sin foco pesquisado en la Unidad de Emergencia Infantil (UEI), evaluado por el equipo de Infectología y que cumpliera con los criterios de inclusión, con tutores responsables y fácil acceso al hospital (Figura 1). La terapia parenteral indicada fue amikacina (dosis 15 $\mathrm{mg} / \mathrm{kg} /$ día) o ceftriaxona (dosis $50 \mathrm{mg} / \mathrm{kg} /$ día), una vez al día, de acuerdo a sospecha de ITU por el hallazgo de sedimento de orina alterado. Se obtuvo el consentimiento informado de los padres para la instalación de un acceso venoso. Se instaló un teflón $\mathrm{N}^{\mathrm{o}} 24$ conectado a una llave de tres pasos en una extremidad, preferentemente la superior (manos o antebrazos) y posterior manejo ambulatorio. El estudio fue aprobado por el comité de ética del hospital.

Los pacientes hospitalizados por indicación del médico tratante de la UEI ingresaron al estudio si cumplían con los criterios antes mencionados.

Se realizó seguimiento de los pacientes hospitalizados

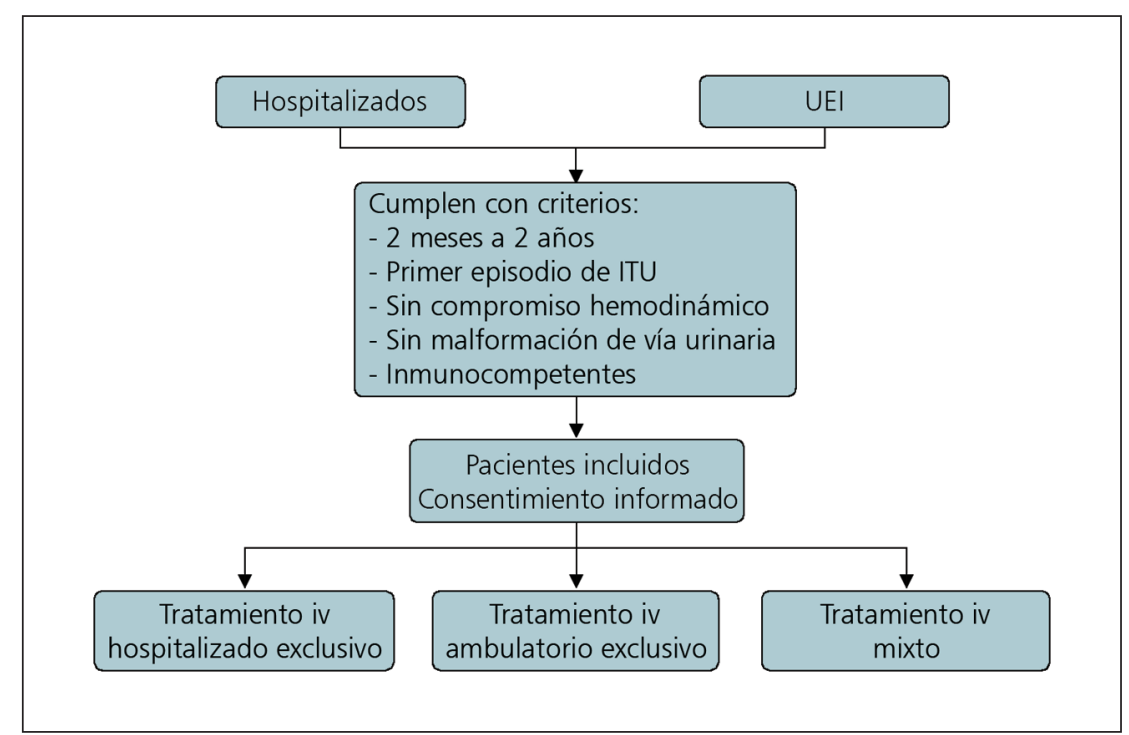

Figura 1. Tratamiento de lactantes con infección urinaria: hospitalizados versus ambulatorios. Flujograma de ingreso de pacientes al estudio. UEI: Unidad de Emergencia Infantil; ITU: infección del tracto urinario.

y ambulatorios, con controles clínicos diarios en la UHT. Una vez obtenido el resultado del urocultivo, y con una evolución favorable (afebril y con buena tolerancia oral), se indicó terapia oral con cefadroxilo (dosis $30 \mathrm{mg} / \mathrm{kg} /$ día) o axetil cefuroxima (dosis $30 \mathrm{mg} / \mathrm{kg} /$ día) o nitrofurantoína (dosis $7 \mathrm{mg} / \mathrm{kg} /$ día) o ciprofloxacina (dosis $20 \mathrm{mg} / \mathrm{kg} /$ día) según informe de susceptibilidad in vitro, completando 7 a 10 días de tratamiento. Se efectuó un urocultivo de control al $5^{\circ}$ día de iniciado el tratamiento y al $5^{\circ}$ día de haber finalizado éste. Todos los pacientes fueron derivados al policlínico de Nefrología del hospital para completar su estudio.

Se registraron los datos demográficos de cada paciente (edad, sexo, peso y talla), los días de tratamiento iv y oral, la adherencia al tratamiento y al seguimiento, los microorganismos aislados y su susceptibilidad in vitro, los efectos adversos inmediatos derivados del antimicrobiano (rash, anafilaxis, náuseas, diarrea, vómitos), las complicaciones asociadas al acceso venoso (flebitis, extravasación, salida accidental, obstrucción), re-hospitalizaciones y mortalidad.

El análisis estadístico se realizó con el programa Stata 9.0 y los datos fueron analizados con $t$ de student, $\chi^{2}$ y test de Fisher según el tipo de variable.

\section{Resultados}

Se enrolaron en el estudio 135 pacientes, 23 recibieron el tratamiento antimicrobiano iv "mixto" (hospitalizado y ambulatorio), por lo que se excluyeron del estudio, 


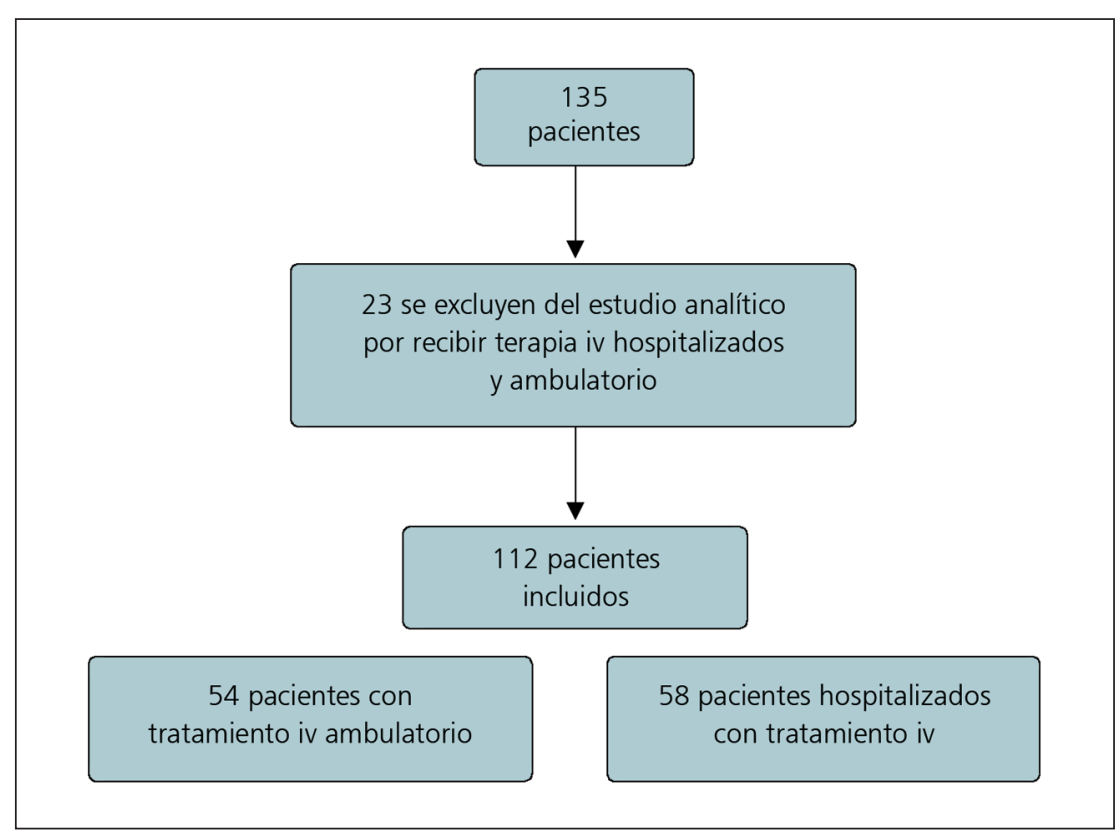

Figura 2. Tratamiento de lactantes con infección urinaria: hospitalizados versus ambulatorios. Distribución de pacientes que cumplieron con criterios de inclusión.

Tabla 1. Tratamiento de lactantes con infección urinaria: hospitalizados versus ambulatorios. Distribución por género y edad promedio de pacientes

\begin{tabular}{|lcccc|}
\hline & n pacientes & $\begin{array}{c}\text { Edad promedio } \\
\text { (meses) }\end{array}$ & DS & Rango \\
\hline Hombres & 42 & $6,33^{*}$ & 2,98 & $2-12$ \\
Mujeres & 70 & $8,5^{*}$ & 4,5 & $2-23$ \\
Total & 112 & 7,7 & 4,1 & $2-23$ \\
\hline$* \overline{\mathrm{x}}$ mujer:hombre $=\mathrm{p} 0,0069$ & & & \\
\hline
\end{tabular}

Figura 3. Tratamiento de lactantes con infección urinaria: hospitalizados versus ambulatorios. Distribución por género ( $p 0,052)$. considerando un total de 112 pacientes para el análisis (Figura 2).

De los 112 pacientes incluidos, $70(62,5 \%)$ fueron mujeres y $42(37,5 \%)$ hombres. La edad promedio para los varones fue de 6,3 meses (DS 2,9 rango entre $2 \mathrm{y}$ 12) y 8,5 meses (DS 4,5 rango de 2 a 23) para el género femenino (p 0,006) (Tabla 1). De este grupo de pacientes, $58(51,8 \%)$ fueron tratados con toda su terapia iv durante su hospitalización y $54(48,2 \%)$ la recibieron en forma ambulatoria exclusiva, no encontrándose diferencias significativas respecto a la distribución según género y edad entre ambos grupos (Figura 3). No se registraron rehospitalizaciones ni ingresos a unidad de paciente crítico. En 3/58 pacientes en el grupo de hospitalizados y 1/54 en el grupo ambulatorio se aisló el mismo microorganismo en el urocultivo y hemocultivo tomado al momento de ingresar al estudio $(\mathrm{p}=0,62)$.

El promedio de días de tratamiento iv para los grupos ambulatorio y hospitalizados fue de 2, 9 días (SD 1,5 rango 1 a 10) y 2,7 días (DS 0,9 rango 1 a 5) respectivamente, sin encontrarse diferencias significativas entre ambos (p 0,22) (Tabla 2).

El principal agente etiológico fue Escherichia coli $-94 \%$ en los hospitalizados, $91 \%$ en el grupo ambulatorio- con similar susceptibilidad in vitro en ambos grupos. Los otros agentes etiológicos aislados representaron un porcentaje mucho menor (Tabla 3).

Durante el seguimiento de los 112 pacientes, se realizó urocultivo de control a los cinco días de tratamiento a 101 $(90,2 \%)$, resultando negativo el $100 \%$ de ellos, tanto para el grupo ambulatorio como hospitalizados. El urocultivo post tratamiento se realizó en $83(74,1 \%)$ pacientes, siendo negativo en $96,4 \%$ de los casos, sin diferencias significativas entre ambos grupos (p 0,93) (Tabla 4). En este control, tres urocultivos resultaron positivos, uno correspondió a $E$. coli de susceptibilidad diferente al primer cultivo, otro resultó negativo al repetirlo y el tercero requirió un nuevo tratamiento antimicrobiano obteniéndose la negativización posterior del urocultivo.

En ninguno de los dos grupos hubo casos fatales, compromiso séptico, absceso renal o complicaciones locales asociadas al acceso venoso.

En el grupo de pacientes que recibió terapia iv hospitalizados, dos casos tenían ITU asociada a patología concomitante $(3,4 \%)$ : un paciente con un cuadro de laringitis y otro con un síndrome diarreico agudo por rotavirus y bronquitis obstructiva por VRS. El promedio de días de estada en el grupo hospitalizado fue 3,5 días (rango: 2-15 días, mediana y moda 2 días), en ellos el costo, exclusivamente por concepto de días-cama, ascendió a $\$ 5.302 .360$, mientras que en el grupo ambulatorio alcanzó a $\$ 1.431 .324$, es decir, un costo $73 \%$ inferior. En caso de haber sido hospitalizado el grupo ambulatorio, habría significado un total de 189 días de estadía, con un 
Tabla 2. Tratamiento de lactantes con infección urinaria: hospitalizados versus ambulatorios. Días de tratamiento intravenoso

\begin{tabular}{|lccc|} 
Modalidad de tratamiento & Promedio & DS & IC $\mathbf{9 5 \%}$ \\
\hline Hospitalizados & $2,7^{*}$ & 0,91 & $2,4-2,9$ \\
Ambulatorio & $2,9^{*}$ & 1,98 & $2,5-3,3$ \\
Total & 2,8 & 1,2 & $2,5-3,0$ \\
\hline${ }^{*}$ p 0,22 & & & \\
\hline
\end{tabular}

Tabla 3. Tratamiento de lactantes con infección urinaria: hospitalizados versus ambulatorios. Microorganismos aislados en el urocultivo inicial

\begin{tabular}{|lrc|}
\hline Microorganismo & n & \% \\
Escherichia coli & 104 & 92,8 \\
Klebsiella pneumoniae & 3 & 2,7 \\
Klebsiella oxytoca & 2 & 1,8 \\
Proteus mirabilis & 2 & 1,8 \\
Enterobacter sp & 1 & 0,9 \\
Total & 112 & \\
\hline
\end{tabular}

costo de $\$ 4.936 .680$. El ahorro en días cama se tradujo en camas disponibles para la atención cerrada de patologías de mayor complejidad (Tabla 5).

En los pacientes que recibieron terapia iv en forma ambulatoria hubo una adherencia de $100 \%$ al tratamiento iv, asistencia para la toma del urocultivo intra-tratamiento de $92 \%$ y al post-tratamiento de $82 \%$. Sólo dos pacientes se hospitalizaron durante el tratamiento por una causa diferente a la ITU.

\section{Discusión}

Comunicamos una experiencia con este nuevo modelo de atención en Pediatría, basado en estándares de calidad similares a otros protocolos de aplicación internacional y demostramos la factibilidad del tratamiento antimicrobiano ambulatorio iv de lactantes febriles con ITU entre dos meses y dos años de edad, con una máxima adherencia, alta efectividad y seguridad, sin encontrar diferencias significativas con los pacientes que recibieron el tratamiento iv inicial hospitalizados. El uso de amikacina y ceftriaxona, en dosis única en niños, es seguro, con adecuado espectro de acción para los microorganismos causantes de ITU en el grupo descrito, logra buenas concentraciones en el parénquima renal, encierra escasas reacciones adversas ${ }^{14-18}$ y ambos están disponibles en nuestro medio. El tiempo de duración del tratamiento iv ( 2,8 días) fue levemente superior a lo descrito en otras experiencias $^{13}$, pero estuvo determinado, en parte, por el tiempo necesario para obtener el aislamiento de microorganismos en cultivos de orina y su susceptibilidad in vitro. La distribución por género, en nuestro medio, fue similar a la descrita en la bibliografía, al igual que el agente etiológico más frecuente $(E$. coli). La recuperación de bacterias en el hemocultivo fue baja, lo que no difiere de otras series publicadas ${ }^{19}$.

El desarrollo del protocolo requirió una buena coordinación entre el lugar donde se realizó el diagnóstico y se inició del tratamiento antimicrobiano y el equipo de
Tabla 4. Tratamiento de lactantes con infección urinaria: hospitalizados versus ambulatorios. Urocultivo de control en pacientes hospitalizados y ambulatorios

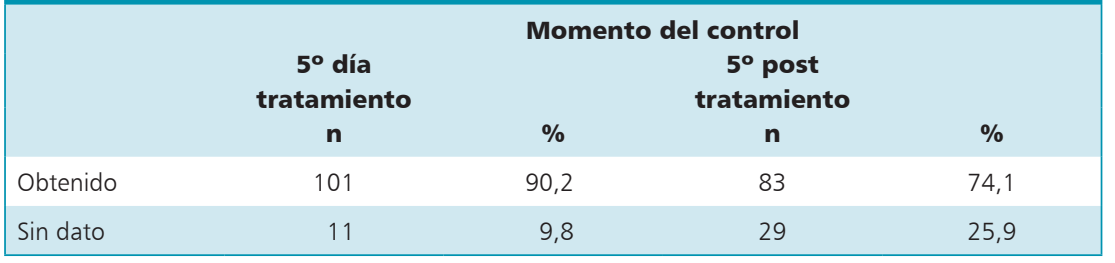

Tabla 5. Tratamiento de lactantes con infección urinaria: hospitalizados versus ambulatorios. Costos día-cama y atención ambulatoria

Modalidad de tratamiento

Hospitalizado

Ambulatorio

(n: 58)

(n: 54)

Promedio días estada

3,5

Promedio días atención iv

2,9

Costo día-cama*

$\$ 5.302 .360$

Costo atención ambulatoria**

$\$ 1.431 .324$

*Arancel FONASA 2007. Modalidad Institucional \$26.120

** Arancel FONASA 2007. Modalidad Institucional \$9.140

manejo ambulatorio implementado por la UHT a cargo del equipo de Infectología pediátrica del CADRS; además, fue condicionado al consentimiento y compromiso de los tutores de los pacientes para las visitas posteriores y manejo en domicilio del acceso venoso.

Es necesario ampliar la experiencia a pacientes con otras patologías, de manera de evitar hospitalizaciones de alto costo emocional para las familias y recursos para el servicio de salud.

Esta modalidad de terapia ambulatoria permite a los pacientes permanecer en su ambiente familiar y evita el riesgo de adquirir una infección asociada a la atención en salud. 
Nuestra experiencia demuestra que la implementación y aplicación de este modelo de manejo es factible y segura, demostrando que con las condiciones de trabajo en el servicio público chileno es posible innovar en gestión de salud con beneficios tanto para el usuario como para el prestador.

\section{Resumen}

Objetivo: Describir la factibilidad, efectividad y seguridad del tratamiento intravenoso (iv) ambulatorio en niños de 2 meses a 2 años con infección del tracto urinario (ITU) y fiebre. Método: Entre abril 2003 y abril 2005 se realizó un estudio prospectivo en pacientes con fiebre sin foco derivados de Emergencia Infantil, finalmente diagnosticados como ITU, estableciéndose dos grupos con tratamiento iv: uno hospitalizado y otro ambulatorio. Se administró amikacina o ceftriaxona según criterio del médico de turno, hasta obtener resultado del urocultivo, y posteriormente se cambió a tratamiento oral. Se controló urocultivo intra y post tratamiento registrándose adherencia, efectividad y complicaciones. Resultados: Se incluyeron 112 pacientes (58 hospitalizados y 54 ambulatorios), con edad promedio de 7,8 meses. El promedio de días de tratamiento iv fue 2,8 días (SD 1,2) sin diferencias significativas entre ambos grupos $2,7+0,91$ días en los internados vs 2,9+1,9 días en los ambulatorios $(\mathrm{p}=0,22)$. En 100\% de ambos grupos de pacientes (en globo 101/101) el urocultivo obtenido al día 5 fue estéril. No hubo complicaciones del tratamiento o sobre la vía venosa. El análisis de costos concluyó que en globo, el manejo ambulatorio representó un 73\% de ahorro económico (1.430.000 pesos chilenos vs 5.300.000 pesos en el sólo rubro de día-cama Conclusiones: El tratamiento iv ambulatorio en pacientes con ITU febril entre 2 y 24 meses fue efectivo, seguro y a un menor costo.

\section{Referencias}

1.- Hellström A, Hanson E, Hansson S, Hjälmas K, Jodal U. Association between urinary symptoms at 7 years old and previous urinary tract infections. Arch Dis Child 1991; 66: 232-4.

2.- Ruscton H G. Urinary tract infections in children: epidemiology, evaluation an management. Pediatr Clin North Am 1997; 44: 1133-69.

3.- Schlagers T. Urinary tract infections in infants and children. Infect Dis Clin North Am 2003; 17 (2): 353-65.

4.- Alper B, Curry S. Urinary tract infections in children. Am Fam Physician 2005; 72 (12): 2483-8.

5.- American Academy of Pediatrics, Committee on Quality Improvement, Subcommittee on Urinary Tract Infection, Practice Parameter: the diagnosis, treatment and evaluation of the initial urinary tract infection in febrile infants and young children. Pediatrics 1999; 103: 843-52.

6.- Lizama M, Luco M, Reichhardt C, Hirsch T. Infección del tracto urinario en un servicio de urgencia pediátrico: frecuencia y características clínicas. Rev Chil Infect 2005; 22: 235-41.

7.- Downs St. Technical report: Urinary tract infections in febrile infant and young children.
Pediatrics 1999; 103 (4): e54.

8.- Shaw K N, Gorelick M, McGowan K L, Yakscoe N M, Schwartz J S. Prevalence of urinary tract infections in febrile young children in the emergency department. Pediatrics 1998; 102 (2): e16.

9.- Ibarra X, Viviani T, Peña A, Cerda J. Síndrome febril sin foco y sospecha de infección bacteriana en niños entre 6 semanas y 36 meses. Rev Chil Pediatr 2008, 79 (4): 388-92.

10.- Benador D, Benador N, Slosman D, Mermillod D, Girardin E. Are younger children at highest risk of renal sequelae after a pyelonephritis? Lancet 1997; 349: 17-9.

11.- Bloomfield P, Hodson E M, Graig J C. Antibiotics for acute pyelonephritis in children (Cochrane review) in The Cochrane library, Oxford, UK: update software; 2003 (3): CD 003772. Review Update in: Cochrane Database Syst Review 2005 (1): CD0037772..

12.- Hoberman A, Wald E R, Hickey R W, Baskin M, Charron M, Majd M, et al. Oral versus initial intravenous therapy for urinary tract infection in young febrile children. Pediatrics 1999; 104: 79-86.

13.- Gauthier M, Chevalier I, Sterescu A, Bergeron S, Brunet S, Taddeo D. Treatment of urinary tract infections among febrile young children with daily intravenous antibiotic therapy at a day treatment center. Pediatrics 2004, 114 (4): 469-76.

14.- Chong D Y, Tan A S, Ng W, Tan-Kendrick A, Balakrishnan A, Chao S M. Treatment of urinary tract infection with gentamicin once or three times daily. Acta Pediatr 2003; 92 : 291-6.

15.- Bartal C, Danon A, Schlaeffer F, Reisenberg K, Alkan M, Smoliakov R. Pharmacokinetic dosing of aminoglycosides: a controlled trial. Am J Med 2003; 114 (3): 194-8.

16.- Krivoy N, Postovsky S, Elhasid R, Ben Arush M W. Pharmacokinetic analysis of amikacin twice and single daily dosage in immunocompromised pediatric patients. Infection 1998; 26 (6): 396-8.

17.- Reids S, Bonadio W. Feasibility of short-term outpatient intravenous antibiotic therapy for the management of infectious conditions in pediatric patients. Am J Emerg Med 2006; 24 (7): $839-42$

18.- Gómez M, Maraqa N, Álvarez A, Rathore M. Complications of outpatient antibiotic therapy in childhood. Pediatr Infect Dis J 2001; 20: 541-3.

19.- Pitetti R D, Choi S. Utility of blood cultures in febrile children with urinary tract infections. Am J Emerg Med 2002; 20: 271-4. 